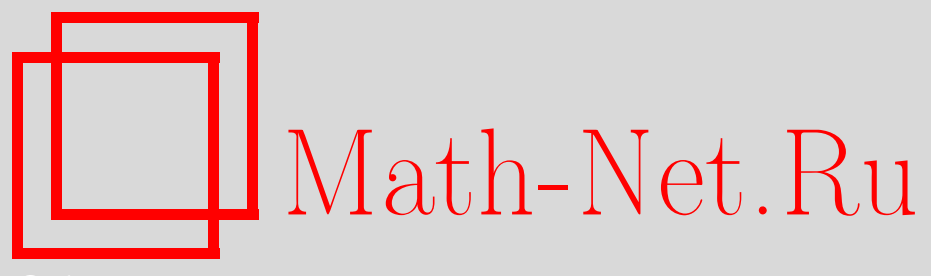

Ж. И. Абдуллаев, И. А. Икромов, Конечность числа собственных значений двухчастичного оператора Шредингера на решетке, ТM $\Phi, 2007$, том 152, номер 3, 502-517

DOI: https://doi.org/10.4213/tmf6106

Использование Общероссийского математического портала Math-Net.Ru подразумевает, что вы прочитали и согласны с пользовательским соглашением http://www . mathnet.ru/rus/agreement

Параметры загрузки:

IP : 54.84 .234 .179

26 апреля 2023 г., $17: 27: 28$

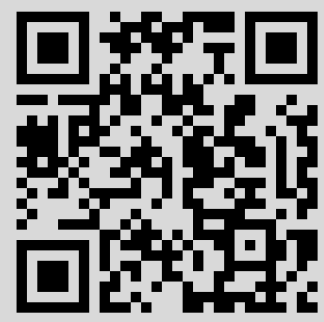




\title{
КОНЕЧНОСТЬ ЧИСЛА СОБСТВЕННЫХ ЗНАЧЕНИЙ ДВУХЧАСТИЧНОГО ОПЕРАТОРА ШРЕДИНГЕРА НА РЕШЕТКЕ
}

\begin{abstract}
В работе рассматривается двухчастичный оператор Шредингера $H(k)$ на $\nu$-мерной решетке $\mathbb{Z}^{\nu}$. Доказана конечность числа отрицательных собственных значений оператора $H(k)$ для широкого класса потенциалов $\hat{v}$.
\end{abstract}

Ключевые слова: гамильтониан, оператор Шредингера, дискретный спектр, принцип Бирмана-Швингера.

\section{1. ВВЕДЕНИЕ}

Условия конечности отрицательного спектра и отсутствия положительных собственных значений двухчастичного непрерывного оператора Шредингера $-\Delta+\lambda V$ приведены в книге [1]. Если $V \leqslant 0$, то число отрицательных собственных значений $N(\lambda)$ - неубывающая функция от $\lambda \in(0, \infty)$. Конечность числа связанных состояний двухчастичных кластерных операторов при малых значениях параметра кластерности была установлена в работе [2], а потом в более общей ситуации в книге [3]. Спектральные свойства двухчастичного дискретного оператора Шредингера $H(k)=H_{0}(k)-V\left(k \in \mathbb{T}^{\nu} \equiv(-\pi, \pi]^{\nu}\right.$ - полный квазиимпульс системы двух частиц) изучены в работах [4]-[7]. В работе [4] рассматривается оператор Шредингера на двумерной решетке и доказывается бесконечность числа собственных значений оператора $H(k)$ при некоторых значениях полного квазиимпульса. При этом, если потенциал $\hat{v}$ принадлежит классу $W(j), j \in\{1,2\}$ (см. ниже формулу (4.5)), то собственные значения $z_{n}(k), n \in \mathbb{Z}$, и соответствующие им собственные функции $f_{n}$, $n \in \mathbb{Z}$, оператора $H(k)$ находятся явно. В работе [5] изучается монотонность каждого собственного значения $z_{n}(k)$ оператора $H(k)$ по координатам $k_{j} \in[0, \pi]$. В работах [6], [7] рассматривается двухчастичный оператор $H(k), k \in \mathbb{T}^{\nu}$, с контактным потенциалом $\hat{v}\left(n_{1}-n_{2}\right)=\mu \delta_{n_{1} n_{2}}$ и устанавливается единственность и невырожденность собственного значения $z(k)$ этого оператора. В работе [8] показано, что двухчастичный оператор $H(k), k \neq 0$, имеет положительное собственное значение,

* Самаркандский государственный университет, Самарканд, Узбекистан. E-mail: jabdullaev@mail.ru, ikromov1@rambler.ru 
лежащее ниже непрерывного спектра, при условии, что $H(0)$ имеет виртуальный уровень в нуле. Некоторые результаты, касающиеся двухчастичного дискретного оператора Шредингера $H(k)$ на трехмерной решетке, докладывались на международной конференции [9].

Следует отметить, что в непрерывном случае “ухудшением” потенциала $V$ можно получить бесконечное число собственных значений вне непрерывного спектра. В этом случае "особенность" невозмущенного оператора в нуле невырожденна и имеет относительно простой вид. Однако в случае дискретного оператора Шредингера особенности невозмущенного оператора могут быть довольно сложными, хотя в некоторых случаях [10] с помощью леммы Морса вид оператора умножения заменой переменных можно свести к сумме квадратов. Как показывают примеры, особенности функций, появляющиеся в зависимости от параметров (квазиимпульса), могут быть неизолированными критическими точками или критическими точками с достаточно большим числом Милнора [11]. Отсюда естественно возникают вопросы. Если потенциал $\hat{v}(n)$ принадлежит некоторому классу последовательностей с убыванием порядка $O\left(|n|^{-\alpha}\right)$, где $\alpha$ - некоторое фиксированное положительное число, то можно ли получить бесконечное число собственных значений с "ухудшением" особенности невозмущенного оператора? Какова связь между особенностью невозмущенного оператора и гладкостью ядра, гарантирующая конечность дискретного спектра возмущенного оператора?

Как известно, в случае одномерного дискретного оператора Шредингера имеются более точные результаты о конечности дискретного спектра [12]. Кроме того, доказано, что если

$$
\sum_{n \in \mathbb{Z}} \hat{v}^{2}(n)=\infty,
$$

то оператор $H(0)$ обязательно имеет бесконечное число собственных значений вне непрерывного спектра. В системе двух фермионов [13] на одномерной решетке получен аналогичный результат о конечности дискретного спектра оператора $H(k)$, который плотно примыкает к результатам Никишина. В работе [13] доказано, что $N(k)$ - число собственных значений оператора $H(k)$ - есть неубывающая функция от $k \in[0, \pi]$ - полного квазиимпульса системы. Описано множество точек разрыва функции $N(k)$ и вычислен скачок $N(k+0)-N(k)$ в точках разрыва. В работе [4] рассмотрен разностный оператор Шредингера $H(k)$ на двумерной решетке и доказано, что оператор $H\left(k_{1}, \pi\right)\left(H\left(\pi, k_{2}\right)\right)$ имеет бесконечное число собственных значений $z_{n}(k), n \in \mathbb{Z}$. Как показано в этой работе, явление бесконечности числа собственных значений связано с неизолированностью точки минимума функции $\varepsilon_{k}$. В работе [9] рассмотрен оператор Шредингера $H(k)$, соответствующий системе двух частиц с одинаковыми массами на трехмерной решетке. Установлено, что для любого $k \in \mathbb{T}^{3} \backslash(-\pi, \pi)^{3}$ существует потенциал $\hat{v}$ такой, что двухчастичный оператор $H(k)$ имеет бесконечное число собственных значений $z_{n}(k)$, накапливающихся к левому краю непрерывного спектра $m(k)$. Кроме того, найдена асимптотическая формула для $z_{n}(k)$ при $n \rightarrow \infty$. Появление бесконечного числа собственных значений также связано с неизолированностью точки минимума функции $\varepsilon_{k}$. 
В данной работе мы получим достаточные условия конечности дискретного спектра оператора Шредингера в зависимости от гладкости потенциала, степень которой зависит от кратности экстремальных значений энергии свободных частиц. Точнее, мы рассмотрим двухчастичный оператор $H(k), k \in \mathbb{T}^{\nu}$, общего вида и докажем конечность числа отрицательных собственных значений этого оператора для широкого класса потенциалов $\hat{v}$.

Следуя известному подходу [14] к исследованию спектра оператора $H(k)$, мы рассмотрим оператор $G(k, z)=r_{0}^{1 / 2}(k, z) V r_{0}^{1 / 2}(k, z), z<0$, где $r_{0}(k, z)$ - резольвента невозмущенного оператора. В случае компактности предельного оператора $G(k, 0):=\lim _{z \rightarrow 0-} r_{0}^{1 / 2}(k, z) V r_{0}^{1 / 2}(k, z)$ мы приводим совершенно элементарное доказательство конечности дискретного спектра оператора $H(k)$. В случае, когда предельный оператор $G(k, 0)$ теряет смысл, мы воспользуемся приемом, анонсированным в работе [15], т.е. представим оператор $V^{1 / 2}$ в виде суммы двух операторов $V_{1}^{1 / 2}+V_{2}^{1 / 2}$, для которой оператор $G_{2}(k, z)=r_{0}^{1 / 2}(k, z)\left(V_{2}^{1 / 2}\right)^{*} V_{2}^{1 / 2} r_{0}^{1 / 2}(k, z)$ компактен при $z \leqslant 0$, а оператор $G_{1}(k, z)=G(k, z)-G_{2}(k, z)$ имеет фиксированный конечный ранг $\ell$ при всех $z \in(-\infty, 0), k \in \mathbb{T}^{\nu}$. При доказательстве в обоих случаях будем использовать принцип Бирмана-Швингера.

\section{2. ОПИСАНИЕ ДВУХЧАСТИЧНОГО ГАМИЛЬТОНИАНА}

Гамильтониан $\widehat{H}$ системы двух квантовых частиц на $\nu$-мерной решетке $\mathbb{Z}^{\nu}$ действует в гильбертовом пространстве $\ell_{2}\left(\left(\mathbb{Z}^{\nu}\right)^{2}\right)$ и имеет вид

$$
\widehat{H}=\widehat{H}_{0}-\widehat{V}_{2},
$$

где свободный гамильтониан $\widehat{H}_{0}$ действует в $\ell_{2}\left(\left(\mathbb{Z}^{\nu}\right)^{2}\right)$ по формуле

$$
\widehat{H}_{0}=-\frac{1}{2 m_{1}} \Delta_{x_{1}}-\frac{1}{2 m_{2}} \Delta_{x_{2}} .
$$

Здесь $m_{1}, m_{2}>0$ означают массы частиц, $\Delta_{x_{1}}=\Delta \otimes I$ и $\Delta_{x_{2}}=I \otimes \Delta$, где $\Delta-$ оператор кинетической энергии отдельной частицы:

$$
(\Delta \hat{\psi})(x)=\sum_{s \in \mathbb{Z}^{\nu}} \hat{\varepsilon}(s) \hat{\psi}(x+s), \quad \hat{\psi} \in \ell_{2}\left(\mathbb{Z}^{\nu}\right),
$$

$\hat{\varepsilon}(s)$ - закон дисперсии. Взаимодействие двух частиц описывается оператором $\widehat{V}_{2}$ :

$$
\left(\widehat{V}_{2} \hat{\psi}\right)\left(x_{1}, x_{2}\right)=\hat{v}\left(x_{1}-x_{2}\right) \hat{\psi}\left(x_{1}, x_{2}\right), \quad \hat{\psi} \in \ell_{2}\left(\left(\mathbb{Z}^{\nu}\right)^{2}\right) .
$$

Относительно вещественнозначных функций $\hat{\varepsilon}$ и $\hat{v}$ предполагается, что

$$
\begin{gathered}
\hat{\varepsilon}(-s)=\hat{\varepsilon}(s), \quad \hat{v}(-s)=\hat{v}(s) \geqslant 0, \quad s \in \mathbb{Z}^{\nu} ; \\
\hat{v}, \hat{\varepsilon} \in \ell_{1}\left(\mathbb{Z}^{\nu}\right) .
\end{gathered}
$$

Условия (2.2) обеспечивают ограниченность операторов $\widehat{H}_{0}$ и $\widehat{V}_{2}$, а вещественность $\hat{v}$ и четность функции $\hat{\varepsilon}$ - самосопряженность этих операторов в $\ell_{2}\left(\left(\mathbb{Z}^{\nu}\right)^{2}\right)$. Из $\hat{v}(s) \geqslant 0$ следует положительность оператора $\widehat{V}_{2}$. 
Переход в импульсное представление осуществляется с помощью преобразования Фурье $\mathcal{F}: \ell_{2}\left(\left(\mathbb{Z}^{\nu}\right)^{2}\right) \rightarrow L_{2}\left(\left(\mathbb{T}^{\nu}\right)^{2}\right)$. Оператор энергии $H=\mathcal{F} \widehat{H} \mathcal{F}^{-1}$ в импульсном представлении коммутирует с группой унитарных операторов $U_{s}, s \in \mathbb{Z}^{\nu}$ :

$$
\left(U_{s} f\right)\left(k_{1}, k_{2}\right)=e^{-i\left(s, k_{1}+k_{2}\right)} f\left(k_{1}, k_{2}\right), \quad f \in L_{2}\left(\left(\mathbb{T}^{\nu}\right)^{2}\right) .
$$

Отсюда следует [1], что существуют разложения пространства $L_{2}\left(\left(\mathbb{T}^{\nu}\right)^{2}\right)$, а также операторов $U_{s}$ и $H$ в прямые интегралы:

$$
L_{2}\left(\left(\mathbb{T}^{\nu}\right)^{2}\right)=\int_{\mathbb{T}^{\nu}} \oplus L_{2}\left(\mathbb{F}_{k}\right) d k, \quad U_{s}=\int_{\mathbb{T}^{\nu}} \oplus U_{s}(k) d k, \quad H=\int_{\mathbb{T}^{\nu}} \oplus \widetilde{H}(k) d k .
$$

Здесь

$$
\mathbb{F}_{k}=\left\{\left(k_{1}, k_{2}\right) \in\left(\mathbb{T}^{\nu}\right)^{2}: k_{1}+k_{2}=k\right\}, \quad k \in \mathbb{T}^{\nu}
$$

$U_{s}(k), s \in \mathbb{Z}^{\nu}, k \in \mathbb{T}^{\nu},-$ оператор умножения на числа $e^{-i(s, k)}$ в пространстве $L_{2}\left(\mathbb{F}_{k}\right)$. Оператор $\widetilde{H}(k), k \in \mathbb{T}^{\nu}$, также действует в $L_{2}\left(\mathbb{F}_{k}\right)$ по формуле

$$
(\widetilde{H}(k) f)(q, k-q)=\left(\frac{1}{m_{1}} \varepsilon(q)+\frac{1}{m_{2}} \varepsilon(k-q)\right) f(q, k-q)-(2 \pi)^{-\nu / 2} \int_{\mathbb{T}^{\nu}} v(q-s) f(s, k-s) d s
$$

и унитарно-эквивалентен оператору $H(k)=H_{0}(k)-V$, называемому оператором Шредингера; унитарность осуществляется с помощью преобразования

$$
u_{k}: L_{2}\left(\mathbb{F}_{k}\right) \rightarrow L_{2}\left(\mathbb{T}^{\nu}\right), \quad\left(u_{k} g\right)(q)=g(q, k-q) .
$$

$H_{0}(k)$ есть оператор умножения на функцию

$$
\varepsilon_{k}(q)=\frac{1}{m_{1}} \varepsilon(q)+\frac{1}{m_{2}} \varepsilon(k-q),
$$

где

$$
\varepsilon(q)=(2 \pi)^{-\nu / 2} \sum_{s \in \mathbb{Z}^{\nu}} \hat{\varepsilon}(s) e^{i(s, q)}
$$

$V$ - интегральный оператор в $L_{2}\left(\mathbb{T}^{\nu}\right)$, порожденный ядром

$$
(2 \pi)^{-\nu / 2} v(p-q)=(2 \pi)^{-\nu} \sum_{s \in \mathbb{Z}^{\nu}} \hat{v}(s) e^{i(s, p-q)} .
$$

Из условий (2.1), (2.2) следует, что функции $\varepsilon$ и $v$ являются четными, вещественнозначными и непрерывными на $\mathbb{T}^{\nu}$.

Обозначим через $m(k)$ и $M(k)$ минимальное и максимальное значения функции $\varepsilon_{k}(q)$, соответственно. Для удобства вместо оператора $H(k)$ мы рассмотрим оператор $H(k)-m(k) I$, заново обозначив его через $H(k)$, т.е.

$$
(H(k) f)(q)=\left(\varepsilon_{k}(q)-m(k)\right) f(q)-(2 \pi)^{-\nu / 2} \int_{\mathbb{T}^{\nu}} v(q-s) f(s) d s .
$$

В силу теоремы Вейля о существенном спектре непрерывный спектр оператора $H(k)$ совпадает со спектром оператора $H_{0}(k)-m(k) I$. Спектр невозмущенного оператора $H_{0}(k)-m(k) I$ чисто непрерывный и совпадает с отрезком $[0, \omega(k)]$. Длина этого отрезка $\omega(k)=M(k)-m(k)$ называется шириной непрерывного спектра оператора $H(k)$. Из самосопряженности оператора $H(k)$ и положительности $V$ вытекает, что $\sigma(H(k)) \cap(\omega(k), \infty)=\varnothing$, поэтому $\sigma_{\text {disc }}(H(k)) \subset(-\infty, 0)$. 
ОПРеДЕЛЕНиЕ. Пусть $\varphi(p)$ - непрерывная вещественнозначная функция на $\mathbb{T}^{\nu}:=$ $(-\pi, \pi]^{\nu}$. Экстремальная точка $p^{0} \in \mathbb{T}^{\nu}$ функции $\varphi(p)$ называется конечнократной, если существуют числа $m>0, c>0$ и окрестность $U\left(p^{0}\right)$ точки $p^{0}$ такие, что для любого $p \in U\left(p^{0}\right)$ справедливо неравенство

$$
\left|\varphi(p)-\varphi\left(p^{0}\right)\right| \geqslant c\left|p-p^{0}\right|^{m},
$$

где $\left|p-p^{0}\right|^{2}=\sum_{i=1}^{\nu}\left(p_{i}-p_{i}^{0}\right)^{2}$. В противном случае экстремальная точка $p^{0}$ называется бесконечнократной. Точная нижняя грань множества чисел $m>0$, для которых выполняется условие (2.5), называется кратностью экстремальной точки $p^{0}$.

Обозначим через $C^{\gamma+0}\left(\mathbb{T}^{\nu}\right)$ пространство определенных на $\mathbb{T}^{\nu}$ непрерывных функций $f$ таких, что для любого мультииндекса $\beta$ с длиной $|\beta| \leqslant[\gamma]$ производная $f^{(\beta)}$ удовлетворяет условию Гельдера с показателем $\{\gamma\}+\tau$ для некоторого $\tau=\tau(f)>0$, где $[\gamma]$ - целая часть, а $\{\gamma\}=\gamma-[\gamma]$ - дробная часть числа $\gamma,|\beta|=\beta_{1}+\beta_{2}+\cdots+\beta_{\nu}$.

Основным результатом работы является следующая

ТЕОРема 2.1. Пусть $\varepsilon_{k}^{-1}(m(k))=\left\{p^{0}\right\}$ и $p^{0}$ является единственной точкой минимума функиии $\varepsilon_{k}$ кратности $2 n$. Тогда для любого $v^{1 / 2}(p)$ из класса $C^{n-\nu / 2+0}\left(\mathbb{T}^{\nu}\right)$ оператор $H(k)$ имеет лищъ конечное число отрицательных собственных значений.

Как отмечено выше, при исследовании дискретного спектра оператора $H(k)$ мы воспользуемся принципом Бирмана-Швингера. Для изложения этого принципа введем некоторые факты и обозначения. Для любого самосопряженного оператора $B$, действующего в гильбертовом пространстве $\mathcal{H}$ и не имеющего существенного спектра правее точки $\mu \in \mathbb{R}$, обозначим через $\mathcal{H}_{B}(\mu) \subset \mathcal{H}$ подпространства такие, что ненулевые элементы $f \in \mathcal{H}_{B}(\mu)$ удовлетворяют неравенству $(B f, f)>\mu(f, f)$, и положим

$$
n(\mu, B)=\sup _{\mathcal{H}_{B}(\mu)} \operatorname{dim} \mathcal{H}_{B}(\mu) .
$$

Число $n(\mu, B)$ совпадает с числом собственных значений оператора $B$, лежащих правее от $\mu$. В работе [8] получена нижняя оценка для числа собственных значений оператора $H(k)$. Число отрицательных собственных значений оператора $H(k)$ не меньше, чем число собственных значений оператора $V$, больших $\omega(k)$, т.е. имеет место неравенство

$$
n(0,-H(k)) \geqslant n(\omega(k), V) .
$$

Как отмечено выше, конечность дискретного спектра оператора связана с поведением кинетической энергии вблизи края непрерывного спектра. В приложении мы исследуем функции в окрестностях экстремальных точек и докажем связь между кратностью экстремальных точек и так называемым числом Милнора [11] для достаточно гладких функций.

\section{3. КОНЕЧНОСТЬ ЧИСЛА СОБСТВЕННЫХ ЗНАЧЕНИЙ}

Для любого $k \in \mathbb{T}^{\nu}$ и $z<0$ определим интегральные операторы

$$
G(k, z)=r_{0}^{1 / 2}(k, z) V r_{0}^{1 / 2}(k, z), \quad Q(k, z)=V^{1 / 2} r_{0}^{1 / 2}(k, z),
$$


где $r_{0}(k, z)$ - резольвента невозмущенного оператора $H_{0}(k)-m(k) I$. Условие $\hat{v}(s) \geqslant 0$ обеспечит положительность оператора $V$. Из условия $z<0$ следует, что $r_{0}(k, z) \geqslant 0$. Через $r_{0}^{1 / 2}(k, z)$ и $V^{1 / 2}$ обозначены положительные квадратные корни операторов $r_{0}(k, z)$ и $V$, соответственно. При условии $(2.2)$ ядро $v^{1 / 2}(p-q)$ интегрального оператора $V^{1 / 2}$ принадлежит $L_{2}\left(\left(\mathbb{T}^{\nu}\right)^{2}\right)$, поэтому $Q(k, z)$ принадлежит классу операторов Гильберта-Шмидта $\Sigma_{2}$. Из представления

$$
G(k, z)=(Q(k, z))^{*} Q(k, z)
$$

следует его положительность и принадлежность классу со следом $\Sigma_{1}$ при всех $k \in \mathbb{T}^{\nu}$ и $z<0$. Кроме того, имеет место принцип Бирмана-Швингера:

$$
n(-z,-H(k))=n(1, G(k, z))
$$

при всех $z<0$. Равенство (3.1) означает, что число собственных значений оператора $H(k)$, лежащих левее $z<0$, совпадает с числом собственных значений оператора $G(k, z)$, бо́льших 1 .

Решение $f$ уравнения Шредингера

$$
H(k) f=z f
$$

и неподвижные точки $\varphi$ оператора $G(k, z)$ связаны соотношениями

$$
f=r_{0}^{1 / 2}(k, z) \varphi, \quad \varphi=\left(H_{0}(k)-(m(k)+z) I\right)^{1 / 2} f .
$$

Так как $\operatorname{Ker} r_{0}^{1 / 2}(k, z)=\{0\}$ при всех $k \in \mathbb{T}^{\nu}, z<0$, то $f$ и $\varphi$ одновременно отличны от нуля.

ЛЕмма 3.1. Число $z<0$ является собственным значением оператора $H(k)$ тогда и только тогда, когда число $\lambda=1$ есть собственное значение оператора $G(k, z)$.

ДокАЗАТЕльство. Пусть ненулевое $f \in L_{2}\left(\mathbb{T}^{\nu}\right)$ является решением уравнения (3.2) эквивалентного уравнению $\left(H_{0}(k)-(m(k)+z) I\right)^{1 / 2} f=r_{0}^{1 / 2}(k, z) V f$. Учитывая соотношения (3.3), получим, что уравнение

$$
\varphi=G(k, z) \varphi
$$

имеет ненулевое решение. И обратно, если уравнение (3.4) имеет ненулевое решение $\varphi \in L_{2}\left(\mathbb{T}^{\nu}\right)$, то функция $f$, построенная по соотношениям (3.3), удовлетворяет уравнению Шредингера (3.2) и принадлежит $L_{2}\left(\mathbb{T}^{\nu}\right)$.

ДоКАЗАТЕЛЬСТво ТЕОРЕмЫ 2.1. Не нарушая общности, предположим, что $p^{0}=0$. Так как $v^{1 / 2} \in C^{n-\nu / 2+0}\left(\mathbb{T}^{\nu}\right)$, то имеет место представление

$$
\begin{gathered}
v^{1 / 2}(p-q)=\sum_{|\alpha|<[n-\nu / 2]} \frac{1}{\alpha !} \frac{\partial^{\alpha} v^{1 / 2}(p)}{\partial q^{\alpha}} q^{\alpha}+\sum_{|\alpha|=[n-\nu / 2]} \frac{1}{\alpha !} \frac{\partial^{\alpha} v^{1 / 2}\left(p-\theta_{\alpha}(q) q\right)}{\partial q^{\alpha}} q^{\alpha}, \\
\theta_{\alpha}(q) \in[0,1],
\end{gathered}
$$


где $\alpha \in \mathbb{Z}_{+}^{\nu}$ - мультииндекс и $|\alpha|=\alpha_{1}+\alpha_{2}+\cdots+\alpha_{\nu}$. Обозначая

$$
\begin{aligned}
& v_{1}^{1 / 2}(p, q)=\sum_{|\alpha| \leqslant[n-\nu / 2]} \frac{1}{\alpha !} \frac{\partial^{\alpha} v^{1 / 2}(p)}{\partial q^{\alpha}} q^{\alpha} \\
& v_{2}^{1 / 2}(p, q)=\sum_{|\alpha|=[n-\nu / 2]} \frac{1}{\alpha !}\left(\frac{\partial^{\alpha} v^{1 / 2}\left(p-\theta_{\alpha}(q) q\right)}{\partial q^{\alpha}}-\frac{\partial^{\alpha} v^{1 / 2}(p)}{\partial q^{\alpha}}\right) q^{\alpha}
\end{aligned}
$$

представим функцию $v^{1 / 2}(p-q)$ в виде сумме $v_{1}^{1 / 2}(p, q)+v_{2}^{1 / 2}(p, q)$. Обозначим через $Q_{i}(k, z), \quad i=1,2, \quad$ интегральный оператор в $L_{2}\left(\mathbb{T}^{\nu}\right)$ с ядром $v_{i}^{1 / 2}(p, q) /$ $\sqrt{\varepsilon_{k}(q)-m(k)-z}$. Тогда

$$
Q(k, z)=Q_{1}(k, z)+Q_{2}(k, z) .
$$

При выполнении условий теоремы 2.1 функция $\partial^{\alpha} v^{1 / 2}(p) / \partial q^{\alpha}$ при $|\alpha|=[n-\nu / 2]$ удовлетворяет условию Гельдера с показателем $\{n-\nu / 2\}+\tau$ для некоторого $\tau>0$. Учитывая, что $\theta_{\alpha}(q) \in[0,1]$ для любого $\alpha,|\alpha|=[n-\nu / 2]$, мы получим, что для функции

$$
a_{\alpha}(p, q)=\frac{\partial^{\alpha} v^{1 / 2}\left(p-\theta_{\alpha}(q) q\right)}{\partial q^{\alpha}}-\frac{\partial^{\alpha} v^{1 / 2}(p)}{\partial q^{\alpha}}
$$

имеет место неравенство

$$
\left|a_{\alpha}(p, q)\right| \leqslant C|q|^{\{n-\nu / 2\}+\tau} \text {. }
$$

Здесь без ограничения общности мы будем предполагать, что $\tau>0,\{n-\nu / 2\}+\tau \leqslant 1$. Из выражений (3.5) и (3.6) получаем, что

$$
\left|v_{2}^{1 / 2}(p, q)\right| \leqslant C|q|^{n-\nu / 2+\tau}
$$

где $C$ не зависит от $p \in \mathbb{T}^{\nu}$. По условию теоремы $2.1 \varepsilon_{k}(q)-m(k) \geqslant C|q|^{2 n}$, поэтому

$$
\frac{\left|v_{2}^{1 / 2}(p, q)\right|^{2}}{\varepsilon_{k}(q)-m(k)} \leqslant \frac{C}{|q|^{\nu-2 \tau}} .
$$

Тогда из неравенства (3.7) следует, что оператор $Q_{2}(k, 0)$ принадлежит классу Гильберта-Шмидта $\Sigma_{2}$. Оператор $Q_{1}(k, z)$ имеет конечный ранг. Обозначим $G_{2}(k, z) \equiv\left(Q_{2}(k, z)\right)^{*} Q_{2}(k, z)$ и $G_{1}(k, z) \equiv G(k, z)-G_{2}(k, z)$. Операторы $G_{1}(k, z)$ и $G_{2}(k, z)$ самосопряженные при $z<0$. Кроме того, предельный оператор $G_{2}(k, 0)$ принадлежит $\Sigma_{1}$, и $G_{2}(k, z)$ равномерно сходится к $G_{2}(k, 0)$ при $z \rightarrow 0$. Оператор $G_{1}(k, z)$ имеет ранг $\ell<\infty$, и число $\ell$ не зависит от $z<0$ и $k \in \mathbb{T}^{\nu}$. Из неравенства Вейля имеем

$$
n(1, G(k, z)) \leqslant n\left(\varepsilon, G_{1}(k, z)\right)+n\left(1-\varepsilon, G_{2}(k, z)\right)
$$

при $0<\varepsilon<1$. Первое слагаемое в правой части этого неравенства не превышает ранга $\ell$ оператора $G_{1}(k, z)$, а второе слагаемое конечно для любого $z<0$ из-за компактности предельного оператора $G_{2}(k, 0)$ и $\lim _{z \rightarrow 0-}\left\|G_{2}(k, z)-G_{2}(k, 0)\right\|=0$. Отсюда и из принципа Бирмана-Швингера (3.1) следует утверждение теоремы.

Из доказательства теоремы 2.1 следует 
Tеорема 3.1. Пусть $\varepsilon_{k}^{-1}(m(k))=\left\{p^{0}, p^{1}, p^{2}, \ldots, p^{s}\right\} u p^{\ell}, \ell \in\{1,2,3, \ldots, s\}, я в-$ ляется точкой минимума функиии $\varepsilon_{k}$ кратности $2 n_{\ell}$. Положим $n_{0}=\max _{1 \leqslant \ell \leqslant s} n_{\ell}$. Тогда для любого $v^{1 / 2}(p)$ из класса $C^{n_{0}-\nu / 2+0}\left(\mathbb{T}^{\nu}\right)$ оператор $H(k)$ имеет лишь конечное число отрицательных собственных значений.

Лемма 3.2. Пусть $\nu \geqslant 3 u \varepsilon_{k}^{-1}(m(k))$ состоит из невырожденных экстремальных точек. Тогда пределъный оператор $Q(k, 0)$ принадлежит классу ГилъбертаШмидта.

ДокАЗАтельство. Очевидно, что каждая экстремальная точка конечного порядка изолирована, поэтому $\varepsilon_{k}^{-1}(m(k))$ содержит конечное число экстремальных точек, скажем, $\varepsilon_{k}^{-1}(m(k))=\left\{p^{1}, p^{2}, \ldots, p^{n}\right\} \subset \mathbb{T}^{\nu}$. Из предположения (2.2) следует, что $v^{1 / 2} \in L_{2}\left(\mathbb{T}^{\nu}\right)$. Пользуясь этим фактом, покажем, что

$$
\int_{\left(\mathbb{T}^{\nu}\right)^{2}}\left|\frac{v^{1 / 2}(p-q)}{\sqrt{\varepsilon_{k}(q)-m(k)}}\right|^{2} d p d q<\infty .
$$

Действительно, делая замену переменных $p-q=p^{\prime}$ в интеграле (3.9), получим

$$
\begin{aligned}
\int_{\left(\mathbb{T}^{\nu}\right)^{2}}\left|\frac{v^{1 / 2}\left(p^{\prime}\right)}{\sqrt{\varepsilon_{k}(q)-m(k)}}\right|^{2} d p^{\prime} d q & =\int_{\mathbb{T}^{\nu}}\left|v^{1 / 2}(p)\right|^{2} d p \int_{\mathbb{T}^{\nu}} \frac{d q}{\varepsilon_{k}(q)-m(k)}= \\
& =\left\|v^{1 / 2}\right\|^{2} \int_{\mathbb{T}^{\nu}} \frac{d q}{\varepsilon_{k}(q)-m(k)} .
\end{aligned}
$$

Так как все экстремальные точки $p^{j} \in \varepsilon_{k}^{-1}(m(k)), j \in\{1,2, \ldots, n\}$, являются невырожденными, то при $\nu \geqslant 3$ последний интеграл сходится.

Теорема 3.2. Пусть выполнены условия леммы 3.2. Тогда оператор $H(k)$ имеет лишь конечное число отрицательных собственных значений, и верно равенство

$$
n(0,-H(k))=n(1, G(k, 0)),
$$

m.е. число отрицательных собственных значений оператора $H(k)$ совпадает $c$ числом собственных значений оператора $G(k, 0)$, бо́лъших 1.

ДокАзАтЕЛьство. В силу леммы 3.2 предельный оператор $G(k, 0)=$ $(Q(k, 0))^{*} Q(k, 0)$ является компактным. Из компактности оператора $G(k, 0)$ и равенства (3.10) следует конечность числа отрицательных собственных значений оператора $H(k)$. Поэтому достаточно доказать равенство (3.10). В работе [8] оно доказано для случая, когда $\nu=3$ и $\Delta$ совпадает с разностным оператором Лапласа на решетке (см. ниже формулу (4.1)). В нашем случае доказательство проводится аналогично.

\section{4. СЛУЧАЙ РАЗНОСТНОГО ОПЕРАТОРА}

В этом разделе мы рассмотрим случай, когда оператор кинетической энергии $\Delta$ совпадает с разностным оператором Лапласа на решетке, т.е.

$$
(\Delta \hat{\psi})(x)=\sum_{j=1}^{\nu}\left[\hat{\psi}\left(x+e_{j}\right)-2 \hat{\psi}(x)+\hat{\psi}\left(x-e_{j}\right)\right], \quad \hat{\psi} \in \ell_{2}\left(\mathbb{Z}^{\nu}\right)
$$


где $e_{j}, j \in\{1,2, \ldots, \nu\},-$ единичные орты в $\mathbb{Z}^{\nu}$. В этом случае функция $\varepsilon(q)$ равна

$$
\varepsilon(q)=\sum_{j=1}^{\nu}\left(1-\cos q_{j}\right)
$$

Функцию $\varepsilon_{k}(p)$, определенную по формуле (2.3), можно представить в виде

$$
\varepsilon_{k}(q)=\frac{\nu}{m_{1}}+\frac{\nu}{m_{2}}-\sum_{j=1}^{\nu}\left[a\left(k_{j}\right) \cos q_{j}+b\left(k_{j}\right) \sin q_{j}\right],
$$

где коэффициенты $a\left(k_{j}\right)$ и $b\left(k_{j}\right)$ равны

$$
a\left(k_{j}\right)=\left(\frac{1}{m_{1}}+\frac{1}{m_{2}}\right) \cos \frac{k_{j}}{2}, \quad b\left(k_{j}\right)=\left(\frac{1}{m_{1}}-\frac{1}{m_{2}}\right) \sin \frac{k_{j}}{2} .
$$

Из равенства (4.2) вытекает следующее представление для функции $\varepsilon_{k}(q)$ :

$$
\varepsilon_{k}(q)=\frac{\nu}{m_{1}}+\frac{\nu}{m_{2}}-\sum_{j=1}^{\nu} r\left(k_{j}\right) \cos \left(q_{j}-p\left(k_{j}\right)\right),
$$

где

$$
r\left(k_{j}\right)=\sqrt{a^{2}\left(k_{j}\right)+b^{2}\left(k_{j}\right)}, \quad p\left(k_{j}\right)=\arcsin \frac{b\left(k_{j}\right)}{r\left(k_{j}\right)} \in[0, \pi] .
$$

Отсюда мы находим, что ширина непрерывного спектра $\omega(k)$ равна

$$
\omega(k)=\sum_{j=1}^{\nu} 2 r\left(k_{j}\right)
$$

В случае одинаковых масс $\left(m_{1}=m_{2}=2 m\right)$ имеем

$$
\varepsilon_{k}(q)=\frac{\nu}{m}-\frac{1}{m} \sum_{j=1}^{\nu} \cos \frac{k_{i}}{2} \cos q_{j}, \quad \omega(k)=\frac{2}{m} \sum_{j=1}^{\nu} \cos \frac{k_{i}}{2} .
$$

Заметим, что при $m_{1}=m_{2}=2 m$ и $k_{i}=\pi$ для любого $p_{i} \in(-\pi ; \pi]$ точка $p=e_{i} p_{i} \in$ $\mathbb{T}^{\nu}$ является бесконечнократной экстремальной точкой для функции $\varepsilon_{k}(p)$. В этом случае экстремальная точка функции $\varepsilon_{k}(p)$ не изолирована.

Очевидно, что $\omega\left(k_{1}, k_{2}, \ldots, k_{\nu}\right)$ есть положительная функция в $(-\pi, \pi)^{\nu}$, четная по каждому $k_{j} \in(-\pi, \pi)$. Заметим, что

$$
\omega(k)=0
$$

тогда и только тогда, когда $m_{1}=m_{2}$ и $k_{1}=k_{2}=\cdots=k_{\nu}=\pi$. Из представления (4.3) и соотношений (4.4) следует

Лемма 4.1. Пусть $m_{1} \neq m_{2}, k \in \mathbb{T}^{\nu}:=(-\pi, \pi]^{\nu}$, или $m_{1}=m_{2}, k \in(-\pi, \pi)^{\nu}$. Тогда функиия $\varepsilon_{k}(p)$ имеет единственный невырожденный минимум.

Теорема 2.1 и лемма 4.1 приводят к следующим теоремам. 
Tеорема 4.1. Пусть $\nu \leqslant 2 u v^{1 / 2}(p) \in C^{1-\nu / 2+0}\left(\mathbb{T}^{\nu}\right)$. Eсли $m_{1} \neq m_{2}, k \in \mathbb{T}^{\nu}$, или $m_{1}=m_{2}, k \in(-\pi, \pi)^{\nu}$, то оператор $H(k)$ имеет лишь конечное число отрицательных собственных значений.

Теорема 4.2. Пусть $\nu \geqslant 3$ и выполняется условие (2.2). Если $m_{1} \neq m_{2}, k \in$ $\mathbb{T}^{\nu}$, или $m_{1}=m_{2}, k \in(-\pi, \pi)^{\nu}$, то оператор $H(k)$ имеет лишь конечное число отрицательных собственных значений.

Известно, что если $\nu=1, m_{1}=m_{2}$, то $\sigma_{\text {disc }}(H(\pi))=\sigma(-V)=\left\{-\hat{v}(n), n \in \mathbb{Z}_{+}\right\}$.

Приведем теперь некоторые факты из работы [4], где рассматривался оператор $H(k)$ с бесконечным числом собственных значений в случае $\nu=2, m_{1}=m_{2}=1$. Обозначим через $W(j), j \in\{1,2\}$, класс потенциалов $\hat{v}$ таких, что

$$
W(j)=\left\{\hat{v}: \operatorname{supp} \hat{v} \subset e_{j} \mathbb{Z} ; \hat{v}\left(n e_{j}\right)>\hat{v}\left((n+1) e_{j}\right), n \in \mathbb{Z}_{+}\right\},
$$

а через $M(j) \subset \mathbb{T}^{2}-$ множество $M(j)=\left\{\left(k_{1}, k_{2}\right): k_{j}=\pi\right\}$. Тогда для любого $(k, \hat{v}) \in M(j) \times W(j)$ оператор $H(k)$ имеет бесконечное число собственных значений $z_{n}(k), n \in \mathbb{Z}_{+}$, вида

$$
z_{n}(k)=\frac{\omega(k)}{2}-\sqrt{\left(\frac{\omega(k)}{2}\right)^{2}+\hat{v}^{2}\left(n e_{j}\right)} .
$$

Более того, из них только $z_{0}(k)$ невырожденное, а остальные $z_{n}(k), n \in \mathbb{N}$, - двукратные собственные значения. Собственным значениям $z_{0}(k)$ и $z_{n}(k), n \in \mathbb{N}$, соответствуют собственные функции

$$
f_{0}(p)=\frac{1}{\varepsilon_{k}(p)-m(k)-z_{0}(k)}, \quad f_{n}^{ \pm}(p)=\frac{e^{ \pm i n p_{j}}}{\varepsilon_{k}(p)-m(k)-z_{n}(k)} .
$$

В работе [9] описано множество $\widehat{M}(j) \subset \mathbb{T}^{3}, j \in\{1,2,3\}$, и класс потенциалов $\widehat{W}(j), j \in\{1,2,3\}$, такие, что если $(k, \hat{v}) \in \widehat{M}(j) \times \widehat{W}(j)$, то оператор $H(k)$ имеет бесконечное число собственных значений $z_{n}(k), n \in \mathbb{Z}$.

ПРИЛОЖКНИЕ

\section{О поведении гладких функций в окрестностях экстремальных точек}

Пусть $U \subset \mathbb{R}^{\nu}$ - некоторая окрестность начала координат, $\varphi(p)$ - непрерывная вещественнозначная функция, определенная на $U$ и имеющая минимум в некоторой точке $p=p_{0}$. Значение функции $\varphi(p)$ в экстремальной точке $p_{0}$ называется экстремальным значением этой функции.

Далее ради упрощения обозначений мы будем считать, что $p_{0}=0$. Согласно известной теореме Ферми, если $\varphi(p)$ - дифференцируемая функция в нуле, то $\nabla \varphi(0)=0$, иными словами, нуль является критической точкой этой функции. Легко показать, что если $\varphi \in C^{2}-$ некоторая функция, имеющая локальный минимум в нуле, и нуль - невырожденная критическая точка, т.е. $\operatorname{Det}\left(D^{2} \varphi(0)\right) \neq 0$, то нуль является экстремальной точкой кратности 2. 
Согласно теореме Лоясевича [16], если $f$ - вещественно-аналитическая функция и $x=0$ - изолированная экстремальная точка, то существует $\mu>0$ такое, что для любой точки $x \in U$ выполняется неравенство $|f(x)-f(0)| \geqslant C|x|^{\mu}$. Иными словами, $x=0$ является экстремальной точкой конечной кратности. Как показывает следующий классический пример, в гладком случае изолированные экстремальные точки могут быть бесконечнократными:

$$
f(x)=\left\{\begin{array}{lll}
e^{-1 / x^{2}} & \text { при } & x \neq 0, \\
0 & \text { при } & x=0 .
\end{array}\right.
$$

В случае выпуклых функций имеется аналогичный более точный результат Шульца [17].

Пусть $f$ - бесконечно-гладкая функция. Функция $f$ называется выпуклой, если для любых точек $x, y \in U \in \mathbb{R}^{\nu}$ (где $U$ - некоторая выпуклая окрестность начала координат) и $\alpha, \beta \in \mathbb{R}$ таких, что $\alpha+\beta=1, \alpha, \beta \geqslant 0$, имеет место неравенство

$$
f(\alpha x+\beta y) \leqslant \alpha f(x)+\beta f(y) .
$$

Гладкая функция $f$ называется функцией конечного типа в точке $x=0$, если для любого единичного вектора $e$ существует натуральное число $N \geqslant 2$ такое, что $D_{e}^{N} f(0) \neq 0$, где $D_{e} f(0)$ производная функции $f$ по направлению вектора $e$ в точке $x=0$. Напомним, что $P$ называется квазиоднородной функцией с весом $\left(1 / n_{1}, \ldots, 1 / n_{\nu}\right)$, если для любого положительного $t$ и $y \in \mathbb{R}^{\nu}$ выполняется равенство

$$
P\left(t^{1 / n_{1}} y_{1}, \ldots, t^{1 / n_{\nu}} y_{\nu}\right)=t P\left(y_{1}, \ldots, y_{\nu}\right) .
$$

ТЕОРЕма П.1 [17]. Если $f$ - выпуклая функиия конечного типа в точке $x=0$ $u|f(0)|+|\nabla f(0)|=0$, то существует вращение $V$ в $\mathbb{R}^{\nu}$ такое, что для функции $f_{1}(y):=f(V y)$ справедливы следующие утверждения:

1) существует квазиоднородный полином $P(y)$ с весом $\left(1 / n_{1}, \ldots, 1 / n_{\nu}\right)$, где $n_{1} \leqslant \cdots \leqslant n_{\nu}-$ некоторые определенные единственным образом четные натуральные числа, такой, что для функиии $f_{1}(y)$ имеет место соотношение

$$
f_{1}(y)=P(y)+R(y)
$$

2) для остатка $R(y)$ справедливо равенство

$$
\lim _{t \rightarrow 0+} \frac{R\left(t^{1 / n_{1}} y_{1}, \ldots, t^{1 / n_{\nu}} y_{\nu}\right)}{t^{1+\varepsilon}}=0,
$$

где $\varepsilon$ - некоторое положительное число;

3) $P(y)$ - выпуклый полином, причем для любого $y \neq 0$ выполняется неравенство $P(y)>0$.

СлЕДСТВиЕ П.1. Если $f$ - выпуклая функция конечного типа и $|f(0)|+$ $|\nabla f(0)|=0$, то точка $y=0$ является экстремальной точкой типа $m$, где $m=$ $\max \left\{n_{1}, \ldots, n_{\nu}\right\}, n_{1}, \ldots, n_{\nu}$ - четные натуральные числа, определенные в теореме П.1. 
ДоКАЗАТЕЛЬСТвО. Без ограничения общности мы можем считать, что $f(y)=$ $P(y)+R(y)$, где $P(y)$ и $R(y)$ - квазиоднородный полином и остаток, определенные согласно теореме П.1.

Заметим, что если $P(y)>0$ - выпуклый квазиоднородный полином с весом $\left(1 / n_{1}, \ldots, 1 / n_{\nu}\right)$, то существуют положительные числа $C_{1}$ и $C_{2}$ такие, что для любого $y \in \mathbb{R}^{\nu}$ выполняется неравенство

$$
P(y) \geqslant C_{1}\left(y_{1}^{n_{1}}+\cdots+y_{\nu}^{n_{\nu}}\right)
$$

и для остаточного члена $R(y)$ в некоторой окрестности нуля имеет место оценка сверху:

$$
R(y) \leqslant C_{2}\left(y_{1}^{n_{1}}+\cdots+y_{\nu}^{n_{\nu}}\right)^{1+\varepsilon} .
$$

Теперь очевидным образом получаем существование окрестности $U$ со свойством

$$
f(y) \geqslant C\left(y_{1}^{n_{1}}+\cdots+y_{\nu}^{n_{\nu}}\right) .
$$

Фактически в некоторой окрестности нуля мы имеем $f(y) \asymp P(y) \asymp y_{1}^{n_{1}}+\cdots+$ $y_{\nu}^{n_{\nu}}$, где символ $\asymp$ означает, что справедливы оценки сверху и снизу с некоторыми константами. Отсюда легко вытекает следствие П.1.

ЗАмЕчаниЕ П.1. Аналогичная оценка снизу для аналитических функций с экстремальной точкой в нуле получена Васильевым [18] в терминах многогранников Ньютона, однако результаты Васильева справедливы лишь для класса аналитических функций общего положения.

Ниже мы покажем связь между кратностью экстремальной точки и числом Милнора. Для полноты изложения приведем соответствующие определения [11].

Пусть $C_{0}^{\infty}\left(\mathbb{R}^{\nu}\right)$ - алгебра ростков бесконечно дифференцируемых функций в нуле и $f$ - бесконечно-гладкая функция, имеющая критическую точку в нуле. Градиентным идеалом, соответствующим функции $f$, называется идеал $I_{\nabla f}$ алгебры $C_{0}^{\infty}\left(\mathbb{R}^{\nu}\right)$, порожденный элементами $\partial f / \partial x_{1}, \ldots, \partial f / \partial x_{\nu}$, т.е.

$$
I_{\nabla f}=\frac{\partial f}{\partial x_{1}} C_{0}^{\infty}\left(\mathbb{R}^{\nu}\right)+\cdots+\frac{\partial f}{\partial x_{\nu}} C_{0}^{\infty}\left(\mathbb{R}^{\nu}\right) .
$$

Локальной алгеброй $Q_{\nabla f}$ градиентого отображения функции $f$ называется фактор-алгебра $Q_{\nabla f}:=C_{0}^{\infty}\left(\mathbb{R}^{\nu}\right) / I_{\nabla f}$.

Локальная алгебра $Q_{\nabla f}$ естественно обладает структурой линейного пространства над $\mathbb{R}$.

Критическая точка 0 гладкой функции $f$ называется конечнократной, если локальная алгебра $Q_{\nabla f}$ является конечномерным линейным пространством над $\mathbb{R}$ размерности $\mu$. Число $\mu$ называется кратностью критической точки или числом Милнора. Мы используем термин “число Милнора", чтобы различать кратности экстремальных и критических точек.

Отметим, что экстремальные точки с конечной кратностью могут быть критическими точками с бесконечным числом Милнора. Например, для функции $f(x)=$

4 Теоретическая и математическая физика, т. 152, № 3, 2007 г. 
$\left(x_{1}^{2}+x_{2}^{2}\right)^{2}$ кратность экстремальной точки 0 равна двум, а соответствующая локальная алгебра $Q_{\nabla f}$ является бесконечномерным линейным пространством. Теперь рассмотрим связь между кратностью экстремальной точки и числом Милнора.

Через $C^{n}(U)$ обозначим пространство $n$ раз непрерывно-дифференцируемых функций на $U$, где $U$ - некоторая окрестность начала координат и $n$ - некоторое фиксированное натуральное число. Пусть $\varphi \in C^{n}(U)$ - функция, имеющая точку минимума в начале координат. Через $m(0)$ обозначим кратность экстремальной точки 0. Если $\varphi \in C^{\infty}(U)$ - функция с критической точкой в нуле, то через $\mu(0)$ обозначается число Милнора для функции $\varphi$ в этой точке.

Для $\varphi \in C^{n}(U)$ через $J^{n}(\varphi)(x)$ [11] обозначается $n$-джет (т.е. отрезок ряда Тейлора порядка $n$ ) функции $\varphi$ в точке 0 .

Известна следующая теорема, доказанная Тужроном [19].

Теорема П.2. Если функиия $\varphi \in C^{\infty}(U)$ имеет критическую точку с числом Милнора $1 \leqslant \mu<\infty$ в нуле, то существует диффеоморфное в окрестности нуля отображсене $g: V \mapsto U$, удовлетворяющее условиям $g(0)=0$ u $\varphi(g(y))=$ $J^{\mu+1}(\varphi)(y)$. Другими словами, $\varphi$ в некоторой окрестности нуля дифбеоморфным отображением приводится к многочлену степени не выше $\mu+1$.

Далее, кратность экстремальных точек оценивается с помощью числа Милнора.

Теорема П.3. Пусть $\varphi$ - вещественнозначная функиия из класса $C^{n+3}(U)$ и $0 \in U-$ экстремальная точка для функции $\varphi$. Если существует бесконечно-гладкая функиия $\psi \in C^{\infty}(U)$ такая, что нуль является критической точкой с числом Милнора $\mu:=\mu(0) \leqslant n$ для функции $\psi$ и $J^{\mu+1}(\varphi)(x)=J^{\mu+1}(\psi)(x)$, то справедливо неравенство $m(0) \leqslant \mu(0)+1$, где $m(0)$ - экстремальная кратность точки 0 для функции $\varphi$. В частности, если $\varphi \in C^{\infty}(U)$ - бункция с экстремальной точкой в нуле и для нее нуль является критической точкой с числом Милнора $\mu(0)<\infty$, то для экстремальной кратности точки 0 справедлива оценка $m(0) \leqslant \mu(0)+1$.

ЗАмечАниЕ П.2. Пусть $f(x)=x^{2 n}$. Тогда число Милнора в нуле $\mu(0)=2 n-1$ и кратность этой экстремальной точки $m(0)=2 n$. Таким образом, в этом случае мы имеем равенство $m(0)=\mu(0)+1$. Этот пример показывает неулучшаемость оценки теоремы.

ДОКАЗАТЕЛЬСТВО ТЕОРЕМЫ П.З. Без ограничения общности мы можем предполагать, что $\varphi(0)=0$. Пусть $P(x)-(\mu+1)$-джет (полином степени не выше $\mu+1$ ) функции $\varphi$ в точке $\alpha=0$. Сначала покажем, что $P(x)$ также имеет минимум в этой точке. Согласно теореме П.2 [19] $\psi$ диффеоморфно эквивалентно $J_{\alpha}^{\mu+1} \psi$. Поэтому мы можем считать $\psi$ полиномиальной функцией, точнее, можно предполагать, что $\psi(x)=P(x)$. Пусть $\mu-$ нечетное число, тогда мы можем написать формулу Тейлора для функции $\varphi$ :

$$
\varphi(x)=q(x)+R(x),
$$

где $q(x)$ - полином степени $\mu+3$ и $R(x)=o\left(|x|^{\mu+3}\right)$. Для фиксированного положительного числа $\varepsilon$ определим вспомогательную функцию $\varphi_{1}(x)=q(x)+\varepsilon|x|^{\mu+3}$. 
Тогда $\varphi_{1}(x)=q(x)+\varepsilon|x|^{\mu+3} \geqslant \varphi(x)$ в некоторой достаточно малой окрестности нуля. Таким образом, $\varphi_{1}(x)$ также имеет локальный изолированный минимум в точке $x=0$. С другой стороны, $\varphi_{1} \in C^{\infty}(U)$. Мы можем применить теорему П.2 к функции $\varphi_{1}$, и она диффеоморфно эквивалентна $P$. Таким образом, и функция $\varphi_{1}$, и $P$ имеют локальные изолированные минимумы в начале координат.

Пусть теперь $\mu$-четное число. В этом случае мы рассмотрим $q(x)-(\mu+2)$-джет функции $\varphi$. Тогда

$$
\varphi(x)=q(x)+R(x)
$$

и $R(x)=o\left(|x|^{\mu+2}\right)$, поэтому вспомогательная функция $\varphi_{1}(x)=q(x)+\varepsilon|x|^{\mu+2}$ также имеет локальный минимум в точке $x=0$. С другой стороны, согласно теореме П.2 $\varphi_{1}$ диффеоморфно эквивалентна $P(x)$. Следовательно, $P(x)$ также имеет локальный минимум в начале координат.

Теперь рассмотрим однопараметрическую деформацию полинома $P(x)$ вида

$$
F_{\varepsilon}(x)=P(x)-\varepsilon x_{1}^{\mu+1},
$$

где $\varepsilon$ - фиксированное вещественное число.

Так как кратность критической точки $x=0$ равна $\mu$, то $x_{1}^{\mu} \in I_{\nabla P}$ [11]. Следовательно, существуют аналитические функции $\left\{h_{k}\right\}_{k=1}^{\nu}$ в начале координат такие, что справедливо тождество

$$
x_{1}^{\mu}=h_{1}(x) \frac{\partial P}{\partial x_{1}}+h_{2}(x) \frac{\partial P}{\partial x_{2}}+\cdots+h_{\nu}(x) \frac{\partial P}{\partial x_{\nu}} .
$$

Поэтому если $\varepsilon$ - достаточно малое число, то справедливо соотношение $I_{\nabla P}=I_{\nabla F_{\varepsilon}}$. Таким образом, при любом фиксированном значении $\varepsilon$ кратность критической точки функции $F_{\varepsilon}$ в нуле равна $\mu$.

Заметим, что $x=0$ является критической точкой конечной кратности для полинома $P$, поэтому существует шар $V \subset C^{\nu}$ с центром в начале координат такой, что для любой точки $x \in \partial V$ (граница шара) справедливо неравенство $|\nabla P(x)|>\delta>0$. Следовательно, существует ненулевое число $\varepsilon$, удовлетворяющее следующим условиям: $F_{\varepsilon}(x)>0$ для всех $x \in \partial V \cap R^{\nu} ;|\nabla P(x)|>|\varepsilon|\left|x_{1}\right|^{\mu}$ для всех $x \in \partial V$. Согласно теореме Руше [20] об отображении многих комплексных переменных отображения $\nabla F_{\varepsilon}(x)$ и $\nabla P(x)$ имеют одинаковое число нулей (с учетом кратности) в $V$. Обе вектор-функции $\nabla F_{\varepsilon}(x)$ и $\nabla P(x)$ имеют нуль одинаковой кратности в начале координат, причем $\nabla P(x)$ не имеет других нулей в $V$, следовательно, $\nabla F_{\varepsilon}(x)$ также не имеет нулей, отличных от $x=0$.

Поэтому при таких фиксированных $\varepsilon$ для любой точки $x \in \bar{V} \cap R^{\nu}$ справедливо неравенство $F_{\varepsilon}(x) \geqslant 0$. Действительно, если существует $x_{0} \in \bar{V} \cap R^{\nu}$ такое, что $F_{\varepsilon}\left(x_{0}\right)<0$, то мы берем $\min _{x \in \bar{V} \cap R^{\nu}} F_{\varepsilon}(x)=m$, и существует $x_{1}$ такое, что $F_{\varepsilon}\left(x_{1}\right)=m$. Так как $F_{\varepsilon}(x)>0$ для всех $x \in \partial V \cap R^{\nu}$, верно $0 \neq x_{1} \in \dot{V} \cap R^{\nu}$, т.е. $x_{1}$ - внутренняя точка множества $V$. Согласно теореме Ферми $\nabla F_{\varepsilon}\left(x_{1}\right)=0$, что противоречит вышеупомянутой теореме Руше.

Таким образом, если $\varepsilon$ - достаточно малое фиксированное число, то существует окрестность нуля $V_{R}=V \cap R^{\nu}$ такая, что при любом $x \in V_{R}$ выполняется неравенство 
$F_{\varepsilon}(x) \geqslant 0$, иными словами, при любом $x \in V_{R}$ имеем

$$
P(x) \geqslant-\varepsilon x_{1}^{\mu+1} \text {. }
$$

Так как $\varepsilon$ - любое достаточно малое вещественное число, то мы можем в качестве $\varepsilon$ взять как достаточно малое положительное число, так и достаточно малое отрицательное. Следовательно, имеется окрестность нуля $V_{R}$ такая, что для любого $x \in V_{R}$ выполняется неравенство

$$
P(x) \geqslant|\varepsilon|\left|x_{k}\right|^{\mu+1}
$$

при $k=1$.

Аналогичные неравенства имеют место при $k=2,3, \ldots, \nu$. Суммируя эти неравенства, получим

$$
P(x) \geqslant \frac{\varepsilon}{\nu}\left(\left|x_{1}\right|^{\mu+1}+\left|x_{2}\right|^{\mu+1}+\cdots+\left|x_{\nu}\right|^{\mu+1}\right) .
$$

Итак, в некоторой окрестности нуля мы имеем неравенство

$$
P(x) \geqslant C|x|^{\mu+1} \text {. }
$$

Так как $\varphi(x)=P(x)+o\left(|x|^{\mu+1}\right)$, то из полученной оценки имеем

$$
\varphi(x) \geqslant C|x|^{\mu+1}
$$

в некоторой окрестности нуля. Отсюда $m(0) \leqslant \mu+1$. Теорема П.3 доказана.

ЗАмечАние П.3. Согласно теореме Васильева [18] число Милнора гладкой функции, имеющей точку экстремума в критической точке, - нечетное. Воспользовавшись этим утверждением, в неравенстве (П.1) мы можем считать $\varepsilon$ отрицательным числом (мы не использовали эту теорему, так как она не упрощает доказательства теоремы П.3).

СлЕДСТвиЕ П.2. Если ч удовлетворяет условиям теоремы П.3 и $P(x)-(\mu+$ 1)-джет этой функици, то в некоторой окрестности нуля справедливо неравенство $\varphi(x) \geqslant P(x) / 2$.

Благодарности. Авторы глубоко признательны рецензенту за ценные замечания. Работа финансировалась ФПФИ АН РУз (гранты № 76-06, 77-06).

\section{Список литературы}

[1] М. Рид, Б. Саймон, Методы современной математической физики. Т. 4. Анализ операторов, Мир, М., 1982.

[2] Ш. С. Маматов, Р. А. Минлос, ТМФ, 79 (1989), 163-179.

[3] R.A. Minlos, A.I. Mogilner, "Some problems conserning spectra of lattice models", Schrödinger Operators, Standard and Nonstandard (Dubna, USSR, 1988), eds. P. Exner, P. Seba, World Sci. Publ., Teaneck, NJ, 1989, 243-257.

[4] Ж.И. Абдуллаев, УзМЖ, 2005, № 1, 3-11.

[5] Ж. И. Абдуллаев, Докл. АН РУЗ, 2005, № 4, 5-9.

[6] Ж. И. Абдуллаев, С. Н. Лакаев, ТМФ, 136 (2003), 231-245. 
[7] P. A. Faria da Viega, L. Ioriatti, M. O'Carroll, Phys. Rev. E (3), 66 (2002), 016130.

[8] S. Albeverio, S. N. Lakaev, J. I. Abdullaev, On the spectral properties of two-particle discrete Schrödinger operators, Preprint. SFB 611. № 170, Univ. Bonn, 2004.

[9] Ж.И. Абдуллаев, “Асимптотика собственных значений двухчастичного оператора Шредингера на решетке", Сборник трудов международной конферениии "Современные проблемы математической физики и информационных технологий”, т. 1 (18-24 апреля 2005, г. Ташкент), 19-23.

[10] М. В. Федорюк, Метод перевала, Наука, М., 1977.

[11] В. И. Арнольд, А.Н. Варченко, С. М. Гусейн-Заде, Особенности дифферениируемых отображений. Классификация критических точек, каустик и волновых фронтов, Наука, М., 1982.

[12] Е. М. Никишин, Тр. сем. им. И. Г. Петровского, 10 (1984), 3-77.

[13] Ж. И. Абдуллаев, ТМФ, 147 (2006), 47-57.

[14] Л. Д. Фаддеев, Математические вопросы квантовой теории рассеяния для системь трех частии, Тр. МИАН, 69, Изд-во АН СССР, М.-Л., 1963.

[15] И. А. Икромов, Ф. Шарипов, Функи. анализ и его прилож., 32:1 (1998), 63-65.

[16] S. Lojasiewicz, Studia Math., 18 (1959), 87-136.

[17] H. Schulz, Indiana Univ. Math. J., 40 (1990), 1267-1275.

[18] В. А. Васильев, Функи. анализ и его прилож., 11:3 (1977), 1-11.

[19] J. C. Tougeron, Ann. Inst. Fourier (Grenoble), 18:1 (1968), 177-240.

[20] Б. В. Шабат, Введение в комплексный анализ. Ч. II. Функиии нескольких переменных, Наука, М., 1985.

Поступила в редакцию 19.06.2006, после доработки 2.12.2006 